\title{
Pour un développement des recherches sur les potentialités de la biodiversité végétale à protéger la santé du consommateur
}

Louis FAHRASMANE*

UMR Qualitrop, Centre INRA Antilles-Guyane, Domaine Duclos, Petit-Bourg, Guadeloupe, F-97170 France

Louis.Fahrasmane@antilles.inra.fr

* Correspondance et tirés à part

Reçu le 14 octobre 2005 Accepté le 28 février 2006

Fruits, 2006, vol. 61, p. 211-222 C) 2006 Cirad/EDP Sciences All rights reserved DOI: $10.1051 /$ fruits:2006018 www.edpsciences.org

RESUMEN ESPAÑOL, p. 222
For a development of research on the plant biodiversity potentialities for protecting human health.

Abstract - Introduction. Today, agri-business products represent a significant and increasing share of food consumed by man. The industrial transformation of the agricultural production has reduced the nutritional density of the processed products. Major agricultural productions. The major world agricultural productions are, to a great extent, of the raw materials for agri-industry. They are mainly cereals and the two major sugar plants (sugarcanes and beets). The space-time availability of the processed products makes it possible to meet calorie needs and to act against hunger. However, for the maintenance of cellular life and physiological equilibrium, it is also necessary to consume micronutrients, of which antioxidants are particularly common and abundant in fruit and vegetables. Fruits, vegetables and health. The consumption of fruit and vegetables, in fresh or little refined forms, is favorable to the protection of the consumer's health. Oils, aromatics and health. Oilseeds and aromatics also contain substances able to contribute to protecting health. Knowledge needs to be developed for a better integration of oils and aromatics in culinary preparations. Antioxidants and biodiversity. Their investigation can be supported by an increase and setting in synergy of: the analytical chemistry and tools, the physiology of plant development, and human nutrition and physiology. The American intertropical zone constitutes a particularly rich area for resources. Conclusion and prospects. It is advisable to seek, in vegetable biodiversity, species of interest for the protection of the consumer's health. It is necessary to carry out an integrative step going from genetic resources to processed products.

Guadeloupe (France) / fruits / vegetables / foods / health / antioxidants / biodiversity

Pour un développement des recherches sur les potentialités de la biodiversité végétale à protéger la santé du consommateur.

Résumé - Introduction. Les produits de l'agro-industrie représentent aujourd'hui une part importante et croissante des aliments consommés par l'homme. La transformation industrielle des productions agricoles réduit la densité nutritionnelle des produits transformés. Productions agricoles majeures. Les productions agricoles mondiales majeures sont pour une bonne part des matières premières pour l'agro-industrie. Ce sont principalement des céréales et les deux plantes sucrières majeures (canne à sucre et betterave). La disponibilité spatio-temporelle des produits transformés permet de répondre aux besoins caloriques et de lutter contre la faim. Or, pour l'entretien de la vie cellulaire et des équilibres physiologiques, il faut aussi consommer des micro-constituants dont les antioxydants, particulièrement communs et abondants dans les fruits et légumes. Fruits, légumes et santé. La consommation des fruits et légumes, sous des formes fraîches ou peu raffinées, est favorable à la protection de la santé du consommateur. Huiles, aromates et santé. Les oléagineux et les aromates contiennent aussi des substances pouvant contribuer à protéger la santé. Des connaissances sont à développer pour une meilleure intégration des huiles et aromates dans les préparations culinaires. Antioxydants et biodiversité. Leur connaissance peut être favorisée par l'essor et la mise en synergie des techniques et outils analytiques, de la physiologie du développement des végétaux, de la nutrition et de la physiologie humaine. La zone intertropicale américaine constitue une aire ressource particulièrement riche. Conclusion et perspectives. Il y a opportunité à rechercher, dans la biodiversité végétale, des espèces d'intérêt pour la protection de la santé du consommateur. Il faut réaliser une démarche intégrative allant de la ressource génétique aux produits transformés.

Guadeloupe (France) / fruits / légume / produit alimentaire / santé / antioxydant / biodiversité 


\section{Introduction}

Aujourd'hui, dans les sociétés modernes et celles des pays en voie de développement, les denrées issues d'opérations de transformations agroindustrielles représentent une part importante et/ou croissante des aliments consommés. Leur qualité fonctionnelle alimentaire, c'est-à-dire leurs propriétés protectrices qui contribuent à réduire la prévalence de certaines maladies, a longtemps été ignorée. De la "fourche à la fourchette ", les critères qui ont longtemps primés sont : le rendement, la conservation, une bonne tenue au transport, la valeur technologique et la praticité de commercialisation et de consommation. Bien souvent, le traitement industriel des ressources agricoles alimentaires mène à une séparation de la fraction énergétique de sa matrice naturelle, ce qui a ouvert la voie à une large industrie de fractionnement et de recomposition alimentaire, qui réduit la capacité des produits transformés à contribuer à protéger la santé.

En matière de qualité des ressources alimentaires, au-delà de l'aspect sanitaire et de la présence de résidus de pesticides, il apparaît pertinent d'intégrer une exigence plus grande autour de la présence et de la disponibilité de substances protectrices de la santé. À cet égard, la densité nutritionnelle, définie par la teneur en éléments nutritionnels intéressants (acides gras, acides aminés essentiels, minéraux, micronutriments) pour un apport calorique donné, est un critère d'appréciation intéressant. Un autre critère de qualité est la teneur en composés antioxydants (polyphénols, caroténoïdes, glucosinolates des crucifères, composés soufrés des alliacées, phyto-oestrogènes, terpènes, etc.), à qui sont reconnues des propriétés de protection de la santé du consommateur.

La disponibilité alimentaire des composés antioxydants, par une nutrition à caractère préventive, apparaît favorable à l'équilibre biologique et psychique de l'homme.

\section{Productions agricoles majeures}

\subsection{Produits alimentaires caloriques}

Les productions agricoles mondiales majeures, dépassant $100 \mathrm{Mt}$ annuelles, sont pour une bonne part des matières premières pour l'agro-industrie. Des céréales (maïs, blé, riz) et deux plantes sucrières (canne à sucre et betterave) représentent plus de $75 \%$ des tonnages récoltés parmi les douze productions majeures (tableau I). L'intensification de la production a reposé sur des variétés à fort rendement, des pratiques culturales intensives, des conditions de récolte, de traitement et de conservation peu optimales pour la densité nutritionnelle des produits consommés. Dans le modèle occidental de consommation, qui tend à se globaliser, l'essentiel de l'énergie des produits alimentaires transformés n'est plus inclus dans la matrice naturelle des ressources agricoles d'origine. Ces produits alimentaires sont d'aspect extérieur flatteur et se révèlent peu savoureux ; leur qualité organoleptique peut reposer sur un relèvement artificiel du goût. Les produits transformés issus des productions agricoles majeures et les huiles assurent l'essentiel de la consommation calorique en alimentation humaine, ils calment la faim. Une part importante des productions céréalières et de leurs coproduits (orge, tourteaux de soja, son, mélasse,) est utilisée pour l'alimentation des animaux.

La réalité alimentaire actuelle est basée sur une certaine logique économique de production-commercialisation-consommation de produits plus ou moins prêts à l'emploi.

Les traitements agro-industriels de première transformation des ressources agricoles permettent d'extraire des fractions, dont celle énergétique, pour produire des ingrédients, pratiquement pas consommés en tant que tel : sucre, huile, amidon, fécule, farine, etc. Ces traitements consistent à :

- Séparer, extraire des molécules (saccharose), des fractions alimentaires et/ou fonctionnelles (semoules, farines, fécules, huiles), à partir de productions agricoles. En bout de filières, ces fractions et extraits sont conditionnés en denrées rendues ainsi plus ou moins disponibles dans l'espace et dans le temps, pour des préparateurs d'aliments (traiteurs, restaurateurs, etc.), les ménages, les éleveurs, des secteurs de production non-alimentaires: papeterie, cartonnerie, pharmacie, etc.

- Transformer, par bioconversion et/ou par des voies physico-chimiques, les 


\begin{tabular}{|c|c|c|c|c|c|c|}
\hline $\begin{array}{l}\text { Rang } \\
\text { mondial }\end{array}$ & Ressource & Genre et espèce & $\begin{array}{l}\text { Production } \\
\text { (t) }\end{array}$ & $\begin{array}{l}\text { Rendement } \\
\left(\mathrm{q} \cdot \mathrm{ha}^{-1}\right)\end{array}$ & $\begin{array}{l}\text { Surface cultivée } \\
\text { (ha) }\end{array}$ & $\begin{array}{l}\text { Type de ressource : } \\
\text { utilisations et / ou produits }\end{array}$ \\
\hline 1 & Canne à sucre & Saccharum sp. & 1393951980 & 652,605 & 20287184 & $\begin{array}{l}\text { Tige tropicale : } \\
76 \% \text { du sucre mondial }\end{array}$ \\
\hline 2 & Maïs & Zea mays & 721379361 & 49,066 & 147022333 & $\begin{array}{l}\text { Céréale : alimentation animale, } \\
\text { amidon, semoulerie }\end{array}$ \\
\hline 3 & Blé & Triticum & 627130584 & 29,065 & 215765044 & $\begin{array}{l}\text { Céréale : alimentations animale } \\
\text { et humaine, semoulerie }\end{array}$ \\
\hline 4 & Riz & Oryza & 605758530 & 40,038 & 151295524 & $\begin{array}{l}\text { Céréale : en l'état et pour } \\
\text { fabrication de boissons }\end{array}$ \\
\hline 5 & Pomme de terre & Solanum tuberosum & 327624417 & 175,857 & 18630196 & $\begin{array}{c}\text { Tubercule légume : alimentation } \\
\text { animale et humaine }\end{array}$ \\
\hline 6 & Betterave sucrière & Beta vulgaris & 249208061 & 444,673 & 5604304 & $\begin{array}{l}\text { Racine sucrière tempérée : } \\
24 \% \text { du sucre mondial }\end{array}$ \\
\hline 7 & Soja & Soja hispida & 204266176 & 22,338 & 91443054 & $\begin{array}{l}\text { Graine légumineuse protéo- } \\
\text { oléagineux : huile tourteaux }\end{array}$ \\
\hline 8 & Manioc & Manihot esculenta & 202648218 & 109,469 & 18511889 & Racine tropicale : tapioca,... \\
\hline 9 & Palmier à huile & Elaeis & 162248628 & 133,924 & 12115010 & Fruit : $2^{\mathrm{e}}$ oléagineux \\
\hline 10 & Orge & Hordeum vulgare & 153624393 & 26,804 & 57313167 & $\begin{array}{c}\text { Céréale : alimentation animale, } \\
\text { brasserie,... }\end{array}$ \\
\hline 11 & Patate douce & Ipomea batatas & 127139553 & 147,513 & 8618866 & $\begin{array}{l}\text { Tubercule tropical: } \\
\text { alimentation humaine }\end{array}$ \\
\hline 12 & Tomate & $\begin{array}{l}\text { Lycopersicon } \\
\text { esculentum }\end{array}$ & 120384017 & 272,255 & 4421734 & $\begin{array}{l}\text { Fruit } 2^{\mathrm{e}} \text { légume : } \\
\text { frais, } 30 \% \text { transformée }\end{array}$ \\
\hline
\end{tabular}

productions agricoles des fractions et extraits : fermentations alcoolique, lactique, boulangerie-pâtisserie, productions de boissons, confiturerie, confiserie, etc.

- Stabiliser des fractions des productions agricoles par les techniques de conservation : stérilisation, pasteurisation, réfrigération, congélations, déshydratation, etc.

- Reformuler : des huiles, des farines, etc.

- Valoriser et traiter les effluents et déchets des sous-produits : tourteaux, mélasse, bagasse, pulpes, eaux résiduaires, etc.

\subsection{Exemple de la canne à sucre}

La transformation sucrière de la canne à sucre (Saccharum officinarum), première récolte mondiale, est un exemple de procédé, ancien, complexe et très élaboré, montrant toute l'ampleur de l'activité agro- industrielle dans l'exploitation par l'homme de productions agricoles. La fabrication du sucre est clairement attestée vers l'an 500 ap. J.-C., à l'ouest du delta de l'Indus et au nord du golf persique, dans le delta du Tigre et de l'Euphrate. Le système de production a été développé en Méditerranée orientale, perfectionné par les croisés après l'an 1000, puis transféré dans les îles atlantiques vers 1450 avant d'être instauré dans les colonies du Nouveau Monde. À cette époque, l'industrie en Europe, à l'exception de la construction navale et de quelques entreprises textiles, reposait en grande partie sur le travail à domicile. La fabrication du sucre présente un caractère industriel précoce dans :

- l'importance de ses moyens humains et mécaniques onéreux,

- son organisation méthodique planifiée et productive, 


\begin{tabular}{|c|c|c|c|c|}
\hline $\begin{array}{l}\text { Rang } \\
\text { mondial }\end{array}$ & Ressource & Genre et espèce & $\begin{array}{l}\text { Production } \\
\text { (t) }\end{array}$ & Remarque \\
\hline 7 & Soja & Soja hispida & 204266176 & Oléo-protéagineux \\
\hline 9 & Palmier à huile & Elaeis & 162248628 & Oléagineux \\
\hline 14 & Graine de coton & Gossypium & 71981922 & - \\
\hline 22 & Noix de coco & Cocos nucifera & 54736517 & - \\
\hline 23 & Colza & Brassica napsus & 46255508 & - \\
\hline 26 & Arachide & Arachis hypogaea & 35723285 & - \\
\hline 32 & Tournesol & Heliantus annuus & 26108358 & - \\
\hline
\end{tabular}

- son fonctionnement saisonnier mais continu,

- la séparation de la production d'avec la consommation, et de l'" ouvrier "qui a été servile jusqu'aux abolitions du $\mathrm{XIX}^{\mathrm{e}}$ siècle d'avec ses outils [1].

Le produit est la molécule de saccharose, un ingrédient purifié, stable, décliné aujourd'hui en une gamme variée : vergeoise, cassonade, candi, glace, roux, blanc, etc. plus ou moins débarrassé d'" impuretés " : minéraux, acides organiques, policosanols, etc. Ces derniers sont recherchés pour leur action réductrice sur le " mauvais cholestérol " [2].

L'utilisation de la canne à sucre et de la betterave sucrière hors de filières industrialisées est quasiment nulle. Ces deux plantes ont de loin les meilleurs rendements agricoles, rendements qui correspondent à la partie usinable récoltée (tableau I). La quantité de sucre extraite est de l'ordre de $10 \%$ de la masse récoltée. Actuellement, le sucre de canne constitue $76 \%$ de la production mondiale de sucre. Dans l'Europe des 15, la moyenne de consommation journalière de sucre (sucre blanc et sucre dans les produits sucrés) est de plus de $93 \mathrm{~g}$ par habitant ${ }^{1}$. C'est dire l'importance de ce produit dans la ration. L'industrie de transformation de la canne à sucre et de ses coproduits génère de nombreux produits commerciaux [3]. Certains sont les plus importants dans leur secteur respectif de production : sucre /

${ }^{1}$ http://www.lesucre.com sucrerie $^{1}$, rhum / eaux de vie $[4,5]$, éthanol / biocarburants $[6,7]$. Le rendement agricole moyen de la canne à sucre (tableau I) en fait une production peu dévoreuse de surface. L'exploitation de cette plante a d'une certaine manière cristallisé les relations entre des groupes humains à l'échelle de la planète, entre le Moyen Âge et le début de l'ère industrielle. En 1773, Bernardin de Saint-Pierre écrivit à propos du café et de la canne à sucre : "... On a dépeuplé l'Amérique afin d'avoir une terre pour les planter ; on a dépeuplé l'Afrique afin d'avoir une nation pour les cultiver." Actuellement, un bel avenir semble promis à la transformation de la canne à sucre dans la production de biocarburants.

\subsection{Des sources de biocarburants}

Dix des douze premières productions agricoles mondiales ainsi que leurs coproduits et déchets ont la particularité d'être des ressources potentielles pour la production de biocarburants [8] :

- bioéthanol à partir de canne à sucre, betterave, maïs, blé, riz, manioc, patate douce, pomme de terre,

- biodiesel à partir d'huiles de palme et de soja, ressources oléagineuses majeures (tableau II),

- biocarburants de nouvelle génération, à partir de polysaccharides provenant de certaines des ressources ci-dessus, mais aussi du bois, en mettant en ouvre des enzymes 
génétiquement modifiées. Les polysaccharides sont transformés par bioconversion en sucres fermentescibles ensuite utilisés comme substrats de fermentation alcoolique.

La canne à sucre et le maïs tiennent d'ores et déjà une place significative dans la production de biocarburants au Brésil, aux États-Unis, en Chine, etc.

\subsection{Un nouveau modèle nutritionnel}

Le modèle nutritionnel qui s'est développé après 1850 était fondé sur une conception du corps assimilé à une machine à brûler des calories. Ce modèle qui privilégiait la viande, laissant une large place aux amidons, aux sucres et aux graisses dont la production industrielle, s'est considérablement développé vers la fin du XIX ${ }^{\mathrm{e}}$ siècle. Dans nos sociétés modernes, le concept des calories est devenu omniprésent dans la réflexion diététique et, à un degré moindre, la répartition des macronutriments (glucide, protéine, lipide) dans la ration, réduisant la prise en compte des effets nutritionnels spécifiques des produits agricoles. Cela a contribué à marginaliser les fruits et les légumes dont on ne voyait pas le réel intérêt énergétique. En 1935, la Commission technique de la ligue des nations émettait l'avertissement suivant : "L'habitude croissante d'une grande consommation de sucre qui tend à diminuer la quantité d'aliments protecteurs dans le régime doit être observée avec attention. "En 1977, des recommandations publiques demandant expressément de manger moins de graisses et de sucre et plus de fruits et de légumes furent adoptées aux États-Unis. L'énergie des aliments doit être accompagnée d'une diversité extraordinaire et subtile de minéraux et microconstituants d'origine végétale, pour entretenir la vie cellulaire et les équilibres physiologiques de notre corps.

\section{Fruits, légumes et santé}

\subsection{Quantités produites}

Si les fruits sont des organes botaniques définis, il n'en est pas de même pour les légumes. Ce sont les parties de plantes pota- gères qui peuvent entrer dans des préparations culinaire : graines, racines et bulbes, tubercules, pousses et capitules, fruits, tiges, feuilles, fleurs, champignons, etc. Les légumes frais ont des propriétés nutritionnelles et fonctionnelles alimentaires globalement comparables à celles des fruits. Beaucoup de légumes sont rendus plus digestibles par la cuisson qui, en dégradant la cellulose et l'amidon, les rend plus accessibles aux enzymes intestinales.

Les tonnages annuels de fruits produits mondialement sont globalement inférieurs d'un facteur dix à ceux des productions majeures (tableaux II et III). Les tonnages de légumes sont globalement inférieurs d'un quart à ceux des productions majeures, quatre légumes étant classés parmi ces productions majeures (tableaux III et $I V$ ). Ces proportions indiquent la place, en terme de quantité, des fruits et légumes dans l'alimentation humaine. Les possibilités de développement de la filière fruits et légumes dans une société moderne soucieuse de protéger la santé de sa population sont considérables. Les fruits et légumes, autres que ceux classés dans les productions majeures, sont destinés essentiellement à l'alimentation humaine.

\subsection{Intérêts nutritionnels et fonctionnels}

Parce que les fruits et les légumes sont riches en eau et pauvres en énergie, leur intérêt nutritionnel a longtemps été sous-estimé. Ils sont pour une part significative consommés frais. C'est de cette manière que le consommateur peut espérer bénéficier au mieux des qualités nutritionnelles et fonctionnelles alimentaires reconnues, et de leurs propriétés organoleptiques : tactiles, visuelles, arômes, saveurs. Au-delà du plaisir que peut procurer la consommation des fruits et légumes frais et de leurs produits dérivés, ils apportent des vitamines, des substances minérales, des oligo-éléments, ainsi que des fibres (pectines, cellulose) et des acides qui ont une action stimulante sur les organes de la digestion. Leur consommation permet une restriction énergétique, sans par ailleurs priver l'organisme de micronutriments protecteurs 
Tableau III.

Les dix premières productions fruitières mondiales annuelles en 2004 (http://faostat.fao.org).

\begin{tabular}{|c|c|c|c|c|c|c|}
\hline $\begin{array}{l}\text { Rang } \\
\text { mondial }\end{array}$ & Ressource & Genre et espèce & $\begin{array}{l}\text { Production } \\
\text { (t) }\end{array}$ & $\begin{array}{l}\text { Rendement } \\
\left(\mathrm{q} \cdot \mathrm{ha}^{-1}\right)\end{array}$ & $\begin{array}{l}\text { Surface cultivée } \\
\text { (ha) }\end{array}$ & Remarques \\
\hline 15 & Banane & Musa sp. & 71343413 & 160465 & 4446044 & $\begin{array}{l}\text { Tropicale, consommée } \\
\text { essentiellement en frais }\end{array}$ \\
\hline 17 & Raisin & Vitacées & 66569761 & 87747 & 7586595 & $\begin{array}{c}\text { Raisin de table frais et } \\
\text { secs, et vins }\end{array}$ \\
\hline 18 & Orange & Citrus sinensis & 62814424 & 174414 & 3601459 & $\begin{array}{l}\text { Consommation en frais, } \\
\text { jus et nectars }\end{array}$ \\
\hline 19 & Pomme & Malus & 61919066 & 119573 & 5178360 & $\begin{array}{l}\text { Frais, boissons : jus, } \\
\text { nectar, cidre, etc. }\end{array}$ \\
\hline 31 & Mangue & Mangifera indica & 26573579 & 72012 & 3690180 & Tropicale \\
\hline 36 & Tangerine, mandarine, clémentine & Citrus reticulata & 22942253 & 131451 & 1745308 & - \\
\hline 40 & Poire & Pirus & 18097558 & 106700 & 1696121 & - \\
\hline 43 & Pêche, nectarine & Prunus persica & 15408553 & 109246 & 1410444 & - \\
\hline 44 & Ananas & Ananas comosus & 15288018 & 181303 & 843231 & Tropicale \\
\hline 46 & Limon et lime & Citrus limon & 12338795 & 153819 & 802163 & - \\
\hline
\end{tabular}

Tableau IV.

Les douze premières productions mondiales annuelles de légume en 2004 (http://faostat.fao.org).

\begin{tabular}{|c|c|c|c|c|c|c|}
\hline $\begin{array}{l}\text { Rang } \\
\text { mondial }\end{array}$ & Ressource & Genre et espèce & $\begin{array}{l}\text { Production } \\
\text { (t) }\end{array}$ & $\begin{array}{l}\text { Rendement } \\
\left(\mathrm{q} \cdot \mathrm{ha}^{-1}\right)\end{array}$ & $\begin{array}{l}\text { Surface cultivée } \\
\text { (ha) }\end{array}$ & Remarques \\
\hline 5 & Pomme de terre & Solanum tuberosum & 327624417 & 175,857 & 18630196 & $\begin{array}{l}\text { Tubercule : alimentation animale } \\
\text { et humaine }\end{array}$ \\
\hline 8 & Manioc & Manihot esculenta & 202648218 & 109,469 & 18511889 & $\begin{array}{l}\text { Racine, production tropicale : } \\
\text { tapioca... }\end{array}$ \\
\hline 11 & Patate douce & Ipomea batatas & 127139553 & 147,513 & 8618866 & $\begin{array}{l}\text { Tubercule tropical : } \\
\text { alimentation humaine }\end{array}$ \\
\hline 12 & Tomate & $\begin{array}{l}\text { Lycopersicon } \\
\text { esculentum }\end{array}$ & 120384017 & 272,255 & 4421734 & Fruit : $30 \%$ sont transformés \\
\hline 13 & Pastèque & Citrullus vulgaris & 94938597 & 275,462 & 3442774 & - \\
\hline 16 & Choux & Brassica spp. & 68169651 & 145,123 & 3170067 & Feuille \\
\hline 24 & $\begin{array}{l}\text { Concombre, } \\
\text { cornichon }\end{array}$ & Cucumis sativus & 40860985 & 168,330 & 2427436 & - \\
\hline 25 & Igname & Dioscorea sp. & 40046149 & 90484 & 4425973 & Tubercule \\
\hline 27 & Plantain (banane) & Musa & 32592554 & 62,632 & 5203812 & Production tropicale \\
\hline 28 & Aubergine & Solanum melongena & 29840793 & 175,466 & 1700655 & - \\
\hline 30 & Melon & Cucumis melo & 27703132 & 210,104 & 1318544 & - \\
\hline 35 & Carotte & Daucus carota & 23997541 & 221,713 & 1081468 & - \\
\hline
\end{tabular}

De nombreuses études épidémiologiques ont mis en évidence le caractère bénéfique de la consommation des fruits et légumes sur le risque de survenue de certaines maladies dites de civilisation. Cette consommation contribue, en effet, à réduire le nom- bre de cas de cancers [9] et de maladies cardio- ou cérébro-vasculaires, et à prévenir des maladies dégénératives telles que la maladie de Parkinson ou encore l'artériosclérose [10]. Le rôle joué par les fruits et légumes dans la prévention de ces maladies 
repose, entre autres (fibres, oligo-éléments), sur les composés antioxydants qu'ils contiennent. On attribue largement aujourd'hui la capacité antioxydante des tissus végétaux aux composés polyphénoliques, aux tanins, aux caroténoïdes (aux propriétés pro-vitaminiques), ainsi qu'à certains oligo-éléments. D'une manière plus large, ces substances participent à l'acceptabilité de l'aliment (couleur et brunissements non désirés) et à sa qualité nutritionnelle. Les potentialités antioxydantes peuvent également faire l'objet d'utilisations non alimentaires : médicinales, paramédicales (cosmétologie, par exemple). La qualité et la teneur en composés phénoliques d'un fruit ou d'un légume dépendent, pour un produit frais, du potentiel génétique, des conditions agro-environnementales de production et de la conservation postrécolte (croissance de la plante productrice, développement et maturation de l'organe, stress divers). Pour un produit transformé, elles dépendront, en plus, des effets des opérations de transformation.

\subsection{Les fruits et légumes majeurs}

Des légumes, comme la pomme de terre et la tomate qui sont des productions agricoles majeures, sont aussi pour une part significative l'objet de transformations alimentaires industrielles, voire non-alimentaires. On estime que $30 \%$ de la production de tomate est transformée industriellement. La production de fécule de pomme de terre et sa transformation sont une industrie très importante qui produit des colles, des produits de lissage du papier, des cartons d'absorbant de couches-culottes, des adjuvants culinaires, des textiles, des liants dans l'industrie et le bâtiment.

Le manioc et la patate douce sont des productions agricoles vivrières majeures, exclusivement tropicales (tableau I), classées parmi les légumes. Leurs utilisations de types traditionnel et artisanal dans leurs zones de production sont encore dominantes. Les produits transformés présentant la praticité des produits modernes sont peu développés. L'étude et la valorisation de propriétés spécifiques pourraient contribuer à développer leur consommation et leur distribution dans de bonnes conditions.
Les agrumes occupent une place importante parmi les fruits. Ce sont plus de $110 \mathrm{Mt}$ qui ont été produites en 2004

La banane dessert, avec $71 \mathrm{Mt}$, est l'espèce fruitière la plus produite mondialement. Elle est consommée en frais. Les bananiers cultivés (dits cultivars) se divisent en deux grands groupes : celui des bananes douces, ou bananes dessert, et celui des bananes à cuire classées parmi les légumes, parmi lesquelles figure la banane plantain. Plus de 120 pays produisent des bananes. Cependant, seulement moins du quart de la production fait l'objet d'un commerce international. Sur les tables occidentales, apparaissent surtout les variétés de bananes desserts cultivées pour l'exportation. La banane dessert saine est un des rares fruits à pouvoir être mangée sans avoir besoin d'aucun instrument, sans se salir les mains, en pouvant ingérer l'intégralité d'une bouchée.

Le fruit du bananier plantain contribue à la sécurité alimentaire de populations des pays défavorisés qui en produisent. Il constitue une ressource alimentaire importante pour plus de $500 \mathrm{M}$ d'habitants des pays tropicaux. Ces fruits sont vitaux pour des millions de paysans qui les produisent, les consomment ou les vendent sur les marchés locaux. Quatre vingt dix pour cent de la production de banane plantain est consommée sur place, notamment dans les pays les plus pauvres d'Afrique, d'Amérique latine et d'Asie. Dans certaines régions, la banane est même la principale denrée cultivée, et la purée de banane y est souvent le premier aliment solide donné aux nourrissons. La consommation se fait souvent sous forme de plat cuit ou de bière. Les connaissances sur la teneur et l'évolution des composés antioxydants dans la banane sont parcellaires.

\subsection{Traitements postrécolte et propriétés}

Les connaissances et méthodes modernes de production fruitière permettent de gérer la physiologie du développement des fruits, le stade de récolte et la vie postrécolte de manière à rendre disponible dans le temps et dans l'espace même les espèces saisonnières. De ce fait, les fruits ne mûrissent pas nécessairement sur pied et, alors, certains 
Tableau V.

Les trois premières productions mondiales de végétaux condimentaires en 2004 (http://faostat.fao.org).

\begin{tabular}{lccccc}
$\begin{array}{l}\text { Rang } \\
\text { mondial }\end{array}$ & Ressource & Genre et espèce & $\begin{array}{c}\text { Production } \\
(\mathrm{t})\end{array}$ & $\begin{array}{c}\text { Rendement } \\
\left(\mathrm{q} \cdot \mathrm{ha}^{-1}\right)\end{array}$ & $\begin{array}{c}\text { Surface cultivée } \\
(\text { (ha) }\end{array}$ \\
\hline 21 & Oignons & Allium cepa & 55153027 & 145,123 & 3049741 \\
34 & Piments, poivrons frais & Capsicum & 24026852 & 190,770 & 1655616 \\
45 & Ail & Allium sativum & 14071355 & 124,557 & 1129714
\end{tabular}

aspects organoleptiques et /ou nutritionnels ne sont pas optimisés.

Certains constituants bénéfiques des fruits et légumes sont diminués par les traitements industriels ou culinaires ${ }^{2}$. Aux échelles domestique, artisanale et industrielle, les fruits sont l'objet de transformations diverses :

- en boissons : jus, nectars, etc.

- en compotes, confitures et confiseries,

- en ingrédients aromatiques, extraits nutritifs, etc.

- en préparations culinaires.

L'approche industrielle est peu adaptée au traitement en permanence de produits frais qui gardent leur complexité et leurs qualités nutritionnelles. De nouvelles techniques de préparation et/ou de stabilisation des productions végétales, qui permettent de mieux préserver les propriétés recherchées des fruits et légumes, sont émergentes : hautes pressions seules ou combinées à la chaleur ou au froid, champs électriques pulsés, etc. " Les recherches en la matière sont motivées par un souci d'altération minimum des qualités des produits, dans une démarche de technologies douces. "[11].

Nous n'attendons plus seulement des aliments qu'ils nous nourrissent et nous procurent du plaisir, mais aussi qu'ils contribuent à la préservation de notre santé. La recherche et l'information dans ce domaine sont devenues un enjeu considérable.

\section{Huiles et aromates pour protéger la santé}

Les fruits et légumes ne sont pas les seules ressources végétales pourvues de propriétés

$\overline{2}$ http://www.wcrf-fr.org favorables à la protection de la santé. Les épices, les herbes et les aromates présentent aussi des propriétés bénéfiques pour la protection de la santé.

Les huiles alimentaires peuvent être supports d'arômes ; elles ont une densité calorique élevée. La production d'huiles vierges ou semi purifiées, à partir de plantes sélectionnées, comme cela a été fait pour l'huile d'olive, pourrait permettre de produire des huiles de grande qualité organoleptique et riches en micro-constituants liposolubles d'intérêt : caroténoïdes, vitamine E, oméga-3, etc. [12]. Les huiles correspondant aux sept plus importantes plantes oléagineuses parmi les productions agricoles (tableau II) sont aussi potentiellement des biocarburants.

Les aromates sont des plantes, fraîches ou séchées, ayant une odeur ou un goût particulièrement prononcé. Les aromates n'ont pas pour seul effet de donner aux aliments un goût plus relevé, ils stimulent aussi toutes les glandes digestives et, de ce fait, l'appétit ; certains d'entre eux sont d'ailleurs pourvus de vertus thérapeutiques.

Les trois premières productions mondiales de plantes condimentaires, selon la base statistique de la FAO, sont l'oignon, les piments et l'ail (tableau V). Les alliacées (ail, oignon, poireau) contiennent des composés soufrés qui leur confèrent des propriétés antibactériennes, inhibent dans l'estomac la conversion des nitrates en nitrites impliqués dans la formation de dérivés cancérigènes [10]. Les piments et poivrons verts ou tournants sont particulièrement riches en vitamine $\mathrm{C}$; on peut en trouver 20 à $30 \mathrm{mg} \cdot \mathrm{mL}^{-1}$ dans le jus des jeunes fruits verts. Ainsi, la vitamine $\mathrm{C}$ a été isolée pour la première fois en 1928 par Albert Szent-Györgyi, à partir de fruits de piments, ce qui lui a valu le prix Nobel de médecine en 1937. On trouve les 
vitamines $\mathrm{A}$ et $\mathrm{E}$ dans les fruits mûrs séchés et les vitamines B1 et B2 y sont également en concentration notable [13].

"Les épices et aromates n'ont pas fait l'objet d'études épidémiologiques comme l'ail, les fruits et les légumes. Des expériences de laboratoire montrent que 37 épices ou aromates frais, parmi les plus courants, ont tous des propriétés antioxydantes et sont de puissants inhibiteurs de tumeurs induites sur des souris, tout spécialement des épices comme le piment de la Jamaïque, la cardamome, la cannelle, le cumin, la graine d'aneth, le gingembre, le poivre, le curcuma, le raifort ainsi que de nombreuses herbes. " [14]. Les concentrations en polyphénols antioxydants mesurées dans des herbes culinaires ont montré des quantités plus fortes que celles observées dans les herbes médicinales ou dans les baies, les fruits et les légumes [15, 16].

Des connaissances sont à développer pour une meilleure intégration des épices et aromates, en quantité et en qualité, dans les préparations culinaires, compte tenu de leur contenu en substances ayant des effets de préservation de la santé. L'examen des usages de certains épices et aromates par certaines sociétés primitives pourrait être alors très informatif.

\section{Antioxydants et biodiversité}

\subsection{Perspectives d'étude des antioxydants}

Autour du développement des techniques et des outils analytiques, de la physiologie du développement des végétaux, et de la nutrition et de la physiologie humaine, il y a une réelle possibilité de mieux appréhender le contenu des fruits et légumes en micro-constituants d'intérêt. La phase de développement des organes et leur vie postrécolte sont à prendre en considération. En aval, des itinéraires technologiques permettant de préserver les micro-constituants et leurs propriétés sont à adapter ou à développer. Une approche pluridisciplinaire s'impose. L'impact physiologique des fruits et légumes est vaste parce qu'il concerne des fonctions aussi diverses que le fonction- nement hépatique, circulatoire, rénal, oculaire, etc.

Les études épidémiologiques confirment les bienfaits des acquisitions alimentaires et culinaires accumulées pendant plusieurs millénaires. Le régime crétois, voire la cuisine crétoise, sont donnés par des nutritionnistes comme modèles. Les fruits et les légumes ne bénéficient pas de communication comparable à celle dont les produits issus de transformations industrielles sont l'objet. L'insuffisance de données analytiques sur le contenu des fruits et des légumes en antioxydants et leur dynamique laisse les filières de production et les consommateurs ignorants et sans repères objectifs.

Il faut élaborer et rechercher des moyens pour mieux connaître les potentialités de la biodiversité à protéger la santé du consommateur. Les connaissances nouvelles générées pourront contribuer à l'élaboration, par le secteur de la transformation agroalimentaire, de voies permettant de commercialiser des produits transformés sous des formes les plus proches possible des ressources fraîches, en complément des choix actuels qui se réduisent souvent à des préparations dégradées lors de leur traitement industriel ou à des produits frais ou surgelés coûteux ou introuvables dans certaines zones. L'enjeu en termes de santé publique est considérable.

Un nouvel âge alimentaire intégrant les progrès fulgurant de la biologie de la physiologie et de la technologie des industries alimentaires paraît indispensable dans le contexte actuel d'attente croissante des consommateurs d'aliments ayant des propriétés de protection de la santé. Dans ce sens, il est d'intérêt général d'identifier et de caractériser le plus grand nombre de ressources de la diversité végétale, ainsi que des produits dérivés et des itinéraires de " la fourche à la fourchette ", permettant de les rendre disponibles aux consommateurs, au mieux de leurs aptitudes à protéger la santé. Dans cette nouvelle dynamique, les ressources connues de la biodiversité tropicale, telle que la banane, appréciées essentiellement pour leurs macro-constituants et leurs propriétés organoleptiques, sont à étudier et à valoriser aussi pour leurs bienfaits en matière de santé. 


\subsection{Les ressources secondaires et mineures}

Les résidus de la transformation industrielle de fruits et légumes peuvent être aussi d'un grand intérêt, comme le montrent les récents travaux de Berardini et Knödler qui se sont intéressés à la valorisation du contenu en pectine et en polyphénols des pelures de la mangue (Mangifera indica), résidus de l'extraction des pulpes [17]. Shin et Leong ont réalisé des travaux sur le contenu des résidus de pressage de la carambole (Averrhoa carambola) en polyphénols antioxydants [18].

À coté des espèces fruitières et légumières cultivées, industrialisées et commercialisées, il existe des espèces secondaires qui n'apparaissent pas dans les statistiques mondiales. Elles sont pour l'instant considérées seulement à l'échelle de leur bassin d'origine et sont assez souvent l'objet uniquement de cueillette. Par exemple, la goyave (Psidium guajava) apparaît particulièrement riche en vitamine C (11 à $980 \mathrm{mg} \cdot 100 \mathrm{~g}^{-1}$ de poids frais), elle n'est pourtant pas valorisée pour cette qualité nutritionnelle. Il en est de même pour le jujube (Ziziphus mauritania) ( $500 \mathrm{mg}$ vitamine $C \cdot 100 \mathrm{~g}^{-1} \mathrm{pf}$ ) qui est cueilli par les enfants et peu transformé, pour la cerise des Antilles ou acerola (Malpighia sp.) [(680 à 4 680) $\mathrm{mg}$ vitamine $\left.\mathrm{C} \cdot 100 \mathrm{~g}^{-1} \mathrm{pf}\right]$ qui est réputée et disponible essentiellement dans ses aires de production, pour le camu-camu (Myrciaria dubia) [(850 à 5 000) mg vitamine $\mathrm{C} \cdot 100 \mathrm{~g}^{-1} \mathrm{pf}$ ] qui est traité dans son aire d'origine, l'Amazone, comme une source naturelle de vitamine $\mathrm{C}$ pour la formulation d'aliments diététiques, etc. [19, 20]. On gagnera à mieux connaître les déterminants de l'évolution du contenu en vitamine $\mathrm{C}$ et en autres composés d'intérêt, à tous les stades de la production lors de l'obtention de produits transformés, de manière à optimiser leur valorisation par des préparateurs culinaires et des consommateurs, mettant en œuvre des modes de préparation déterminés et adaptés.

\subsection{Intérêt de la zone méso américaine}

La zone intertropicale américaine est particulièrement riche en biodiversité végétale.
Elle est à prospecter dans la perspective d'accroître, en quantité et en qualité, la disponibilité des ressources ayant des propriétés favorables à la préservation de la santé par l'alimentation. Aujourd'hui, $40 \%$ des espèces végétales alimentaires utilisées dans le monde sont d'origine américaine, la cuisine précolombienne n'a pas connu une extension comparable. D'après Boudan [14], parmi les 250000 espèces végétales de la planète, 100000 seraient américaines, alors que 12000 seulement constitueraient la flore européenne. L'Amérique tropicale a apporté une large contribution à l'alimentation mondiale [21]. Boudan [14] écrit en 2004 : "Pour une revue rapide de l'Amérique latine, mentionnons ... des tubercules (pommes de terre, patate douce, topinambour, achira, anu, arracacha, arrow-root, jicama, maca, manioc, mashua, oca, ullucu, uncucha, yacon, etc.). La liste des légumes américains est plus réduite: tomate, tomate verte, chayotte, citrouille, potiron, courge, gourde, buacatai, buaotzontle, paico... La liste des épices et aromates n'est pas non plus très longue : piment, poivre dit d'Amérique, poivre de la Jamaïque, achiote, vanille. Mais la quantité de fruits est considérable : ananas, annone, avocat, canneberge (au nord), cerise verte, cerises des Antilles et de Guyane, cherimolia, ciruela, corossol, figue de Barbarie, fraises du Chili et de Virginie, framboise (au nord), fruit de la passion, goyave, grenadille, guanabana, kaki de Virginie, lulo, mamey, myrtille, guava, papaye, poire de Quito, pommecannelle, sapote, tomate d'arbre, etc., sans oublier quelques fruits secs tels que noix de cajou, cacao, hickory et noix de pécan. "

\section{Conclusion et perspectives}

L'urbanisation croissante et les transformations connexes escomptées dans les modalités de production et de commercialisation des denrées alimentaires se traduisent par un allongement de la chaîne alimentaire et par certains risques d'introduction ou d'aggravation de dangers d'origine alimentaire.

Si la cuisine est une tentative, fondée sur l'expérience cumulée, pour définir les règles qui optimisent l'utilisation de ressources 
nutritionnelles dans un double objectif alimentaire et santé, on comprend pourquoi les populations qui ont gardé un régime traditionnel fondé sur la cuisine des produits directement issus de l'agriculture sont souvent en meilleure santé et ont globalement une plus grande longévité que celles qui s'alimentent de produits transformés. Il y a un courant fort de désengagement de la sphère domestique de l'activité culinaire. L'Occident est en voie d'étendre au monde entier un modèle d'alimentation dans lequel les objectifs poursuivis par la cuisine domestique seraient laissés aux mécanismes de marché. Dans les zones urbanisées du monde, la cuisine quotidienne réalisée avec des produits frais s'efface au profit de la consommation de préparations et de repas " prêts-à-l'emploi ", produits par l'industrie.

Il y a opportunité de rechercher, dans la biodiversité végétale, des espèces d'intérêt pour la protection de la santé du consommateur. Il faut réaliser une démarche intégrative, tenant compte de la dimension génétique, des conditions agro-pédo-climatiques, de la physiologie du développement et de la maturation, ainsi que de l'évolution postrécolte des composés d'intérêt, en fonction des traitements technologiques et/ou culinaires. Les différentes parties récoltées des ressources agricoles ainsi que les coproduits et sous-produits sont à considérer. Les acquis d'une telle démarche pourront contribuer à faire évoluer l'agro-alimentaire vers la distribution d'un plus grand nombre d'aliments issus d'itinéraires de production permettant la proximité avec leur complexité naturelle. Les structures opérationnelles de recherche, pour ce faire, doivent être au plus près des lieux de production, pour intégrer au mieux la réalité biologique et physiologique des ressources cibles.

\section{Références}

[1] Mintz S., Sucre blanc, misère noire, Nathan, Paris, France, 1991.

[2] Arruzazabala M.D., Carbajal D., Mas R., Molina V., Castano G., Gamez R., Effects of D-003, a new compound purified from sugarcane wax, on platelet aggregation in healthy volunteers: a randomised, double-blind clinical study, Clin. Drug Invest. 23 (2003) 107118.

[3] Patureau J.M., By-products of the cane sugar industry, Elsevier Sci. Publ. Co., AmsterdamOxford-New-York, 1988.

[4] Laboissière J.-L., Le rhum, un festival de cannes international, Rayon Boissons 93 (2002) 30-31.

[5] Fahrasmane L., Ganou-Parfait B., De la canne au rhum, Inra Ed., Paris, France,1997.

[6] Bungay H.R., Confessions of a bioenergy advocate, Trends Biotechnol. 22 (2) (2004) 67-71.

[7] Theil S., The next petroleum, Newsweek, Aug. 8, 2005 issue, 42-48.

[8] Claassen P.A.M., Van Lier J.B., Lopez Contreras A.M., Van Niel E.W.J., Sijtsma L., Stams A.J.M., De Vries S.S., Weusthuis R.A., Utilisation of biomass for the supply of energy carriers, Appl. Microbiol. Biot. 52 (1999) 741755.

[9] Gerber M., Alimentation méditerranéenne et cancers, Sci. Aliment. 24 (2004) 267-277.

[10] Grolier P., Borel P., Scalbert A., Remesy C., Les phytomicronutriments, in : Basdevant A., Laville M., Lerebours E, Traité de nutrition clinique de l'adulte, Éd. Flammarion, Paris, France, 2001.

[11] Bimbenet J.J., Trystram G., Le point des recherches en génie des procédés alimentaires, Ind. Aliment. Agric. 122 (3) (2005) 8-18.

[12] Rémésy C., Que mangerons-nous demain ? Éd. Odile Jacob, Paris, France, 2005.

[13] Pitrat M. Foury C., Histoires de légumes, Inra Éd., Paris, France, 2003.

[14] Boudan C., Géopolitique du goût, PUF, Paris, France, 2004

[15] Zheng W. Wang, S.Y., Antioxydant activity and phenolic compounds in selected herbs, J. Agric. Food Chem. 49 (2001) 5165-5170.

[16] Atoui A.K., Mansouri A., Boskou G., Panagiotis K., Tea and herbal infusions: their antioxydant activity and phenolic profile, Food Chem. 89 (2005) 27-36.

[17] Berardini N., Knödler M., Scieber A., Carle R., Utilization of mango peels as a source of pectin and polyphenolics, Food Sci. Emerg. Technol. 6 (4) (2005) 442-452. 
[18] Shui G., Leong L., Residue from star fruit as valuable source for functional food ingredient and antioxydant nutraceuticals, Food Chem. 97 (2) (2006) 277-284

[19] Rodrigues R.B., De Menezes H.C., Cabral L.M.C., Dornier M., Reynes M., An Amazonian fruit with a high potential as a natural source of vitamin C: the camu-camu (Myrciaria dubia), Fruits 56 (2001) 345-354.
[20] Gomez P., Reynes M., Dornier M., Hebert J-P., La cerise des Antilles: une exceptionnelle source de vitamine $C$ naturelle, Fruits 54 (1999) 247-260.

[21] Garine Y., Les modes alimentaires : histoire de l'alimentation et des manières de table, in: Histoire des mœurs, 1, Encyclopédie de la Pléiade, Gallimard, Paris, France, 1990.

Para un desarrollo de las investigaciones de las potencialidades de la biodiversidad vegetal para proteger la salud del consumidor.

Resumen - Introducción. Los productos de la industria agrícola representan hoy una parte importante y en auge de los alimentos consumidos por el hombre. La transformación industrial de las producciones agrícolas reduce la densidad nutricional de los productos transformados. Producciones agrícolas mayores. Las principales producciones agrícolas mundiales cuentan con una parte considerable de las materias primas de la industria agrícola. Se trata esencialmente de cereales y de las dos principales plantas de azúcar (azúcar de caña y azúcar de remolacha). La disponibilidad espacio-temporal de los productos transformados permite responder a las necesidades calóricas así como luchar contra el hambre. Ahora bien, para el mantenimiento de la vida celular y para los desequilibrios fisiológicos, también es necesario consumir micro-constituyentes cuyos antioxidantes, particularmente comunes y abundantes en frutas y en verduras. Frutas, verduras y salud. El consumo de frutas y verduras, bajo diversas formas frescas o poco refinadas es favorable para la protección de la salud del consumidor. Aceites, aromatizantes y salud. Los oleaginosos y los aromatizantes contienen también sustancias que pueden contribuir a proteger la salud. Se deben desarrollar conocimientos para una mejor integración de los aceites y de los aromatizantes en las preparaciones culinarias. Antioxidantes de la biodiversidad. Su conocimiento puede favorecerse por la reactivación y la puesta en sinergia de las técnicas y de las herramientas analíticas, de la fisiología del desarrollo de los vegetales, de la nutrición y de la fisiología humana. La zona intertropical americana constituye un área de recurso particularmente rica. Conclusión y perspectivas. Existe la oportunidad de investigar, en la biodiversidad vegetal, especies de interés para la protección de la salud del consumidor. Es necesario realizar una acción integrada que abarque desde el recurso genético hasta los productos transformados.

Guadalupe (Francia) / frutas / hortalizas / alimentos / salud / antioxidantes / biodiversidad 